\title{
The Inhibition of the Expression of the Small Rho GTPase Rac1 Induces Differentiation With no Effect on Cell Proliferation in Growing Human Adult Keratinocytes
}

\author{
Ekaterina Nikolova, ${ }^{1,2}$ Vanio Mitev, ${ }^{2}$ Frédéric Minner, ${ }^{1}$ Christophe F. Deroanne, ${ }^{3}$ and Yves Poumay $^{1}$ * \\ ${ }^{1}$ Cell and Tissue Laboratory, URPHYM, University of Namur (FUNDP), B-5000 Namur, Belgium \\ ${ }^{2}$ Department of Chemistry and Biochemistry, Medical University of Sofia, 1431 Sofia, Bulgaria \\ ${ }^{3}$ Laboratory of Connective Tissues Biology, CBIG/GIGA Research Center, University of Liège, \\ B-4000 Sart Tilman, Belgium
}

\begin{abstract}
Rac1 is a Rho subfamily small GTPase which is highly expressed in epidermal keratinocytes. In mice the significance of Rac1 for the maintenance of the epidermis has been controversial. In keratinocytes from human origin, the role of Rac1 in the control of growth/differentiation is still obscure. In this study we used RNA interference to induce specific inhibition of Rac1 expression in cultured human keratinocytes and analyzed the consequences on proliferation and differentiation. We found that the autocrine proliferation of keratinocytes is unaltered by Rac1 silencing. However, the suppression of Rac1 induced premature differentiation as revealed by the expression of markers (keratin 10, involucrin), but the involved mechanism is independent of the activity of p38 mitogen-activated protein kinase. Rather, we found that the effects of Rac1 silencing on keratinocytes differentiation are concomitant with negative regulation of the Ser62/Thr58 phosphorylation on the transcription factor c-myc, a mechanism known to control post-translational stability of the c-myc protein. Thus, in growing human keratinocytes, Rac1 could impede the expression of premature differentiation markers, probably by exerting positive control on c-myc activity and its binding to specific promoters. J. Cell. Biochem. 9999: 1-8, 2007. @ 2007 Wiley-Liss, Inc.
\end{abstract}

Key words: normal human adult keratinocytes; Rac1; proliferation; differentiation; c-myc; MAPK

Keratinocytes, the major epidermal cell type, proliferate and subsequently differentiate to ensure the continual renewal of the protective outermost layer of the skin. Any disturbance in the balance between proliferation and differentiation of keratinocytes can disrupt the epidermal homeostasis and be the cause of skin disorders [De Luca et al., 1994; Rao et al., 1996; Iizuka et al., 2004]. Thus, understanding the complex signaling mechanisms underlying the physiological regulation of epidermal keratinocytes remains a major challenge of skin biology.

The institution at which the work was performed: Cell and Tissue Laboratory, URPHYM, University of Namur (FUNDP), B-5000 Namur, Belgium.

Grant sponsor: FRFC; Grant number: 2.4506.01.

*Correspondence to: Yves Poumay, $\underline{\mathrm{Cell}}^{\mathrm{Q} 1}$ and Tissue Laboratory, URPHYM, University of Namur (FUNDP), B-5000 Namur, Belgium. E-mail: yves.poumay@fundp.ac.be Received 20 March 2007; Accepted 30 May 2007

DOI $10.1002 / j$ cb. 21455

Published online 00 Month 2007 in Wiley InterScience (www.interscience.wiley.com).

(c) 2007 Wiley-Liss, Inc.
Small Rho GTPases exert regulatory functions on specific signaling events in keratinocytes. For instance, Rho function on distinct transduction pathways to coordinate control on cytoskeletal reorganizations and growth/differentiation processes. The three best studied Rho family members, Rho-A, -B, and -C have been found to promote the establishment of epithelial adhesion and simultaneously exert strict control on the epidermal differentiation process [Braga et al., 1997; Grossi et al., 2005]. The selective inhibition of Rho-A, $-\mathrm{B}$, and $-\mathrm{C}$ by bacterial toxin $\mathrm{C} 3$ causes premature differentiation of growing keratinocytes from mouse and human origins [Grossi et al., 2005].

Rac1 is another Rho family member expressed in epidermal keratinocytes. Similarly to the closely related Rho-A, -B, and -C, Rac1 is involved in the dynamic changes during keratinocytekeratinocyte adhesion [Braga et al., 1997]. The role of Rac1 in the control of keratinocytes has been recently studied in vivo by two research groups using mice models with restricted epidermal deletion of Rac1 [Benitah et al., 2005; 
Chrostek et al., 2006], however their results were contradictory: according to Benitah et al. [2005], epidermal deletion of Rac1 increases proliferation of basal keratinocytes together with an increased expression of the differentiation marker keratin 10 (K10), mediated by an increased c-myc expression and activity; according to Chrostek et al. [2006], keratinocyte-restricted deletion of Rac1 gene in mutant mice does not affect the normal proliferation rate of basal keratinocytes, does not change the expression of the differentiation markers, K10 and loricrin, and does not alter c-myc expression. The likely reasons for these contradictory results may be due to the different genetic backgrounds of the mice used in the two studies, or to the different methods utilized for Rac1 deletion in skin.

In our present in vitro study, we applied the technique of RNA interference (RNAi) in order to achieve a specific Rac1 protein suppression [Wang and Zheng, 2007] in cultured normal human adult keratinocytes (NHAK). Then, we studied the effects of Rac1 silencing on the rate of DNA synthesis, on the expression of differentiation markers (K10 and involucrin) and on the expression and phosphorylation of the c-myc transcription factor. Our results indicate that Rac1 silencing induces premature differentiation without affecting the proliferation of the cultured human keratinocytes. Concomitantly, we report that the suppression of Rac1 does not alter c-myc expression but its phosphorylation at Ser62/Thr58 involved in posttranslational control of c-myc protein [Sears et al., 2000], suggesting that Rac1 negatively controls keratinocyte differentiation, maybe by interaction with c-myc.

\section{MATERIALS AND METHODS}

\section{Reagents}

Primary antibodies were purchased from the following manufacturers: mouse anti-Rac1 (23A8) from Upstate cell signaling solutions, The Netherlands; mouse anti-RhoA (sc-418) from Santa Cruz Biotechnology, CA; mouse anti-Cdc42 from BD Transduction Laboratories, Belgium; mouse anti-Human Cytokeratin 10, Dako Cytomation, Denmark; mouse antiHuman Cytokeratin 14, Novocastra laboratories Ltd, UK; rabbit anti-Human Involucrin, Harbor Bio-Products, MA; mouse anti-phosphoERK1/2 (Thr202-Tyr204), rabbit anti-phosphop38 (Thr180-Tyr182), and rabbit anti-p38 MAP
Kinase from Cell Signaling; Danvers, MA; rabbit anti-phospho- JNK1/2 (Thr183-Tyr185) from Biosource International, Inc.; mouse anti c-myc (Invitrogen Life Technologies); rabbit anti-phospho-c-Myc (Thr58/Ser62) from Cell Signaling; mouse anti- $\beta$-actin (AC-15) from Sigma, St. Louis, MI. Secondary antibodies were goat anti-mouse-HRP and goat anti-rabbitHRP, both from Dako Cytomation. Methyl$\left[{ }^{3} \mathrm{H}\right]$-thymidine is from MP Biomedicals, Inc., Irvine, CA.

\section{Cell Cultures}

Normal human adult keratinocytes (NHAK) were isolated from skin explants obtained during abdominal plastic surgery from 25 to 45 years old healthy donors as described [Poumay et al., 1999]. Third and fourth passages keratinocytes were seeded at density $7 \times 10^{3}$ cells $/ \mathrm{cm}^{2}$ and were maintained in serum-free EpiLife Medium (Cascade Biologics, Inc., UK) with added growth supplement (Human Keratinocytes Growth Supplement, Cascade Biologics, Inc.) and antibiotics. The cells were incubated at $37^{\circ} \mathrm{C}$ and $5 \% \mathrm{CO}_{2}$ till reaching $50-60 \%$ confluence, then switched to autocrine growth conditions by excluding the growth factors from the medium. The cells were fed every other day with fresh medium. All the experiments were performed on cells cultured under autocrine growth conditions.

\section{siRNA Transfection}

21-nucleotide long siRNAs were chemically synthesized, desalted, deprotected, and purified by polyacrylamide-gel electrophoresis (PAGE) (Eurogentec, Belgium). The 5'-GAUAAAGACACGAUCGAGA-3' and 5'-UCUCGAUCGUGUCUUUAUC-3' oligoribonucleotides were used to inhibit Rac1. The sense and antisense oligoribonucleotides were annealed at concentration $100 \mu \mathrm{M}$ in $50 \mathrm{mM} \mathrm{NaCl}, 1 \mathrm{mM}$ EDTA, $10 \mathrm{mM}$ Tris-HCl, $\mathrm{pH}$ 7.5. As a control, we used the siRNA negative control, a perfectly unique sequence which does not match to any sequence in the human genome, recommended by the manufacturer (cat \#OR-0030-NEG05, Eurogentec). Transient transfections were carried out using Oligofectamine (Invitrogen Life technologies) and manufacturer's instructions. The cells were split in 24 well plates 1 day before transfection. On the next day when reaching $50 \%$ of culture confluence, keratinocytes were 
transfected in medium without antibiotics with Rac1-specific siRNA or siRNA control duplex at $100 \mathrm{nM}$ final concentration. The silencing of Rac1 was assayed 3 days after the transfection by at least three independent experiments.

\section{DNA Synthesis Assay: $\left[{ }^{3} \mathrm{H}\right]$ Thymidine Incorporation}

Keratinocytes were split in 24 well plates, at a density which allows cells to reach $50 \%$ of confluence on the next day, then the transfection was performed as above. Forty eight hours later, the cells were switched to autocrine growth conditions for $18-20 \mathrm{~h} .\left[{ }^{3} \mathrm{H}\right]$-Thymidine at final concentration $1 \mu \mathrm{Ci} / \mathrm{ml}$ was added to the culture medium $4 \mathrm{~h}$ before harvesting and analyzing the cells. Briefly, cells were washed, then treated successively with ice-cold PBS, 10\% TCA (twice) and 5\% TCA (three times) and lysed in $500 \mu \mathrm{l} 1 \mathrm{~N} \mathrm{NaOH}$ and $0.1 \%$ SDS. The rate of proliferation was measured as the radioactivity incorporated into DNA using a Beckman Liquid Scintillation Counter (Beckman Coulter, Inc., Fullerton, CA).

\section{Western Blot Analyses}

For Western blotting, the cells transfected with Rac1-specific siRNA or siRNA control duplex were washed in ice-cold PBS, harvested and solubilized in lysis buffer $(20 \mathrm{mM}$ Tris- $\mathrm{HCl}$ (pH 6.8), $2 \%$ SDS, $10 \%$ saccharose, $50 \mathrm{mM}$ ditiothreitol, $0.1 \%$ bromophenol blue). Equal amounts of total protein were separated on $7.5 \%, 10 \%$, or $12.5 \%$ polyacrylamide gels depending on the effective range of separation of the gel and the respective molecular weight of the analysed proteins ( $7.5 \%$ gel for involucrin$68 \mathrm{kDa} ; 10 \%$ gel for total and phospho c myc$64 \mathrm{kDa}, \mathrm{K} 10-56.5 \mathrm{kDa}, \mathrm{K} 14-50 \mathrm{kDa}$, phospho-ERK 1/2—42/44 kDa; phospho JNK1/2$46 / 54 \mathrm{kDa}$; total and phospho p38-43 kDa; beta actin-42 kDa; $12.5 \%$ gel for RhoA-24 kDa; Cdc42-22 kDa, Rac1-21 kDa). The proteins were transferred to polyvinylidene difluoride (PVDF) membranes (Millipore, Bedford, MA) and immunoblotting analyses with specific primary antibodies and peroxidase-conjugated secondary antibodies diluted 1:1,000 were carried out using the ECL technique (Roche Molecular Biochemicals, Indianapolis, IN). To confirm equivalent protein loading, blots were probed with an antibody to $\beta$-actin or to total p38.

\section{Real-Time RT-PCR Analyses}

Total RNA was isolated from the transfected cells by applying RNeasy Mini Kit (Qiagen, France). One microgram of total RNA was used for cDNA preparation by using SuperScriptTM II RNase H Reverse Transcriptase (Invitrogen Life Technologies) following the manufacturer's protocol. After cDNA preparation, $1 \mathrm{ng}$ was mixed with $10 \mu \mathrm{l}$ Power Sybr Green PCR Master Mix (Applied Biosystems) and specific oligonucleotide primers (Sigma-Aldrich, UK), at $300 \mathrm{nM}$ final concentration, for Rac1 (sense GCTAAGGAGATTGGTGCTGTAAAA, antisense CTGCTCGGATCGCTTCGT), K14 (sense CGATGGCAAGGTGGTGTC, antisense GGGTGAAGCAGGGTCCAG), K10 (sense AATCAGATTCTCAACCTAACAAC, antisense CTCATCCAGCACCCTACG), and involucrin (sense TGAAACAGCCAACTCCAC, antisense TTCCTCTTGCTTTGATGGG) for real-time quantitative PCR analysis. The results are expressed as specific relative mRNA levels measured in regard of the house-keeping gene 36B4 mRNA expression. For the measurement of 36B4 expression, the following specific oligoribonucleotide primers were used: sense ATCAACGGGTACAAACGAGTC and antisense CAGATGGATCAGCCAAGAAGG.

\section{RESULTS}

\section{siRNA-Mediated Specific Reduction of Rac1 Protein Level in Cultured Normal Human Keratinocytes}

Wishing to investigate the functions of Rac1 in epidermal keratinocytes, we decided to evaluate the consequences induced in cultured NHAK by a selective inhibition of Rac1 expression. A specific Rac1 siRNA (hereafter designated as siRac1) was introduced into cultured human epidermal keratinocytes by carrying out a transient transfection mediated by oligofectamine. This transfection produced an efficient Rac1 protein suppression in epidermal keratinocytes (Fig. 1A) when compared with the level of Rac1 detected after the same transfection performed with control siRNA (hereafter designated as siRNA control). Furthermore, our siRac1 duplex showed highly specific inhibitory action on Rac1 protein expression without affecting other closely related Rho subfamily members: Cdc42 and RhoA (Fig. 1A). When observed by phase-contrast microscopy 3 days 

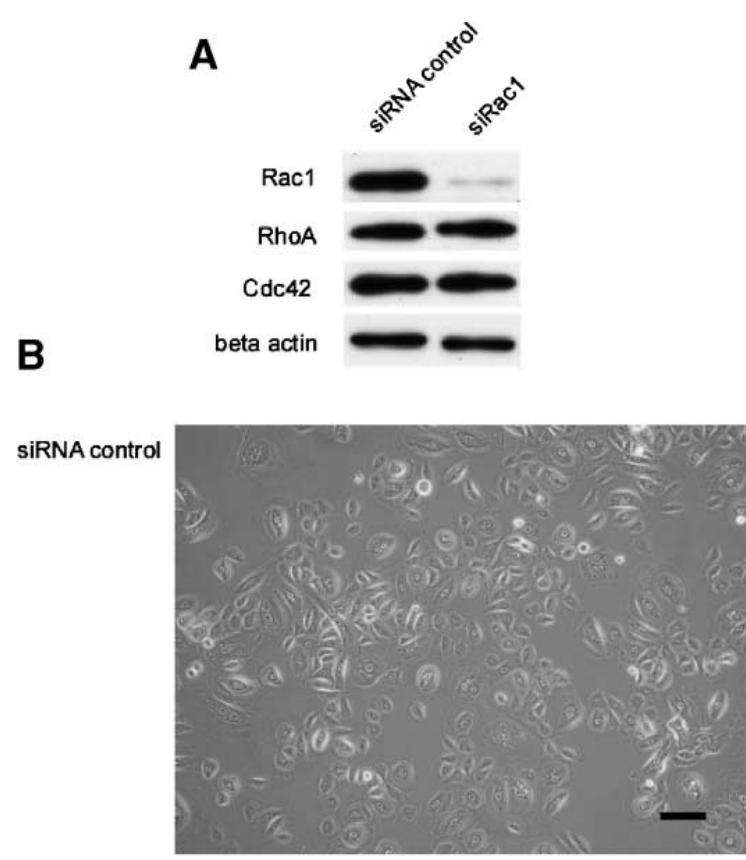

siRac1

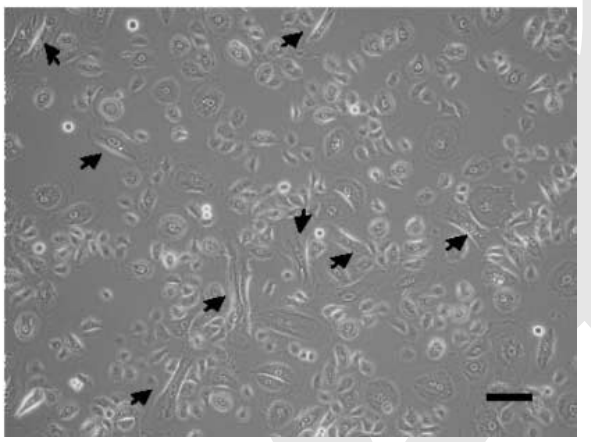

Fig. 1. Rac1 protein expression is efficiently suppressed by specific small interfering RNA in NHAK and is accompanied by an altered morphology in several cells. A: A test for specificity and efficiency of siRac1 was fulfilled by Western blot analyses. NHAK were transfected with siRac1 or siRNA control as described in Materials and Methods. Three days after transfection, treated cells were harvested, their total protein was extracted and subjected to Western blot analyses with primary antibodies specific for Rac1, Cdc42 and RhoA. Beta-actin levels were determined as a protein loading control. Representative data of three independent experiments. B: Rac1-specific small interfering RNA in parallel with non-specific control RNA were introduced in NHAK by carrying out a transient transfection as it was described in Materials and Methods. Three days after transfection, NHAK were screened by phase-contrast microscopy in order to detect alteration due to siRNA-mediated Rac1 silencing. Arrows point elongated shape keratinocytes frequently observed after siRac1 treatment. Scale bars, $100 \mu \mathrm{m}$.

after transfection, keratinocytes transfected with siRac1 revealed in several cells an elongated shape phenotype (Fig. 1B, arrows) less frequently observed in keratinocytes transfected with siRNA control (Fig. 1B), suggesting that Rac1 is involved in normal cell shape in keratinocytes.

\section{Rac1 Silencing Does not Alter DNA Synthesis in Growing Cultured Keratinocytes}

In order to detect whether Rac1 is playing a role in the control of NHAK proliferation, we performed transient transfection with siRac1 or siRNA control. Then, 3 days later, the cells were allowed to incorporate radioactive $\left[{ }^{3} \mathrm{H}\right]$-thymidine. The amount of incorporated $\left[{ }^{3} \mathrm{H}\right]$-thymidine determined per $\mu \mathrm{g}$ of protein revealed no significant change (9-11\% inhibition) in the rate of keratinocytes DNA-synthesis after siRNA-mediated Rac1 silencing, compared to the control (Fig. 2).

\section{Rac1 Silencing Influences the Expression Level of Differentiation Markers, but not the Expression of a Basal Marker}

We applied siRNA specific inhibition to achieve selective Rac1 suppression in keratinocytes, then we examined the consequences on the expression of basal (K14) and differentiation (K10 and involucrin) markers. Both real time RT-PCR and Western blot analyses confirmed upregulation of differentiation markers at mRNA and protein levels after Rac1 silencing compared to the siRNA control (Fig. 3A,B). Concomitantly, the expression of the basal K14 marker remained unchanged.

\section{(3H)Thymidine incorporation}

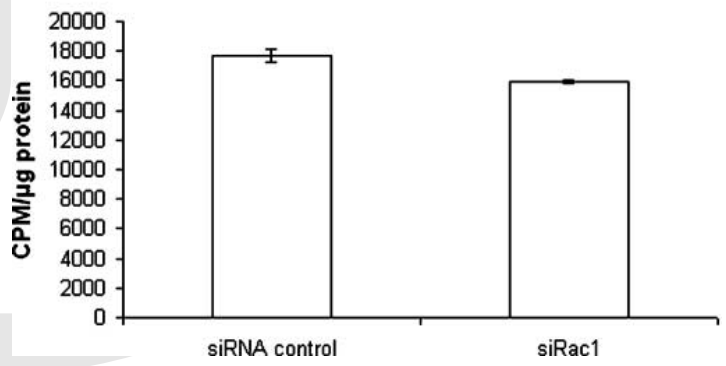

Fig. 2. The inhibition of Rac1 expression has no significant effect on the rate of DNA synthesis in NHAK. NHAK were transiently transfected with siRac1 or siRNA control as it was described in Materials and Methods. Forty eight hours after the transfection, the cells were switched to autocrine growth conditions for 18-20 h. Four hours before cell analysis, $1 \mu \mathrm{Ci} /$ $\mathrm{ml}$ of $\left[{ }^{3} \mathrm{H}\right]$-thymidine was added to the culture medium. The rate of cell proliferation was measured by determining the radioactivity incorporated into DNA. Data are mean \pm SEM of three independent experiments. 


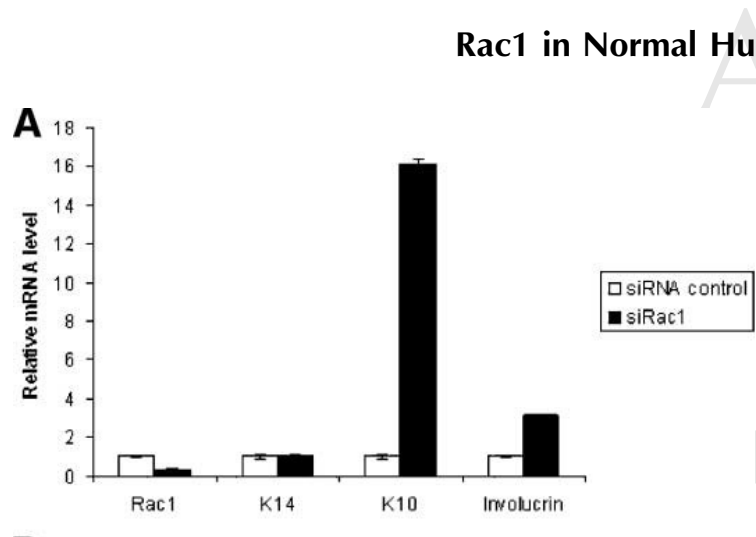

B

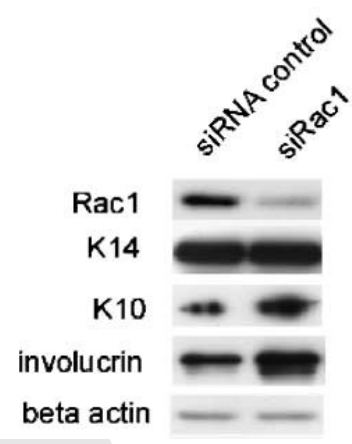

Fig. 3. Rac1 silencing induces the expression of differentiation markers as detected both at mRNA and protein levels. NHAK were transiently transfected with siRac1 or siRNA control as described in Materials and Methods. Three days after cell transfection, the expression of proliferative marker, K14 and differentiation markers, $\mathrm{K} 10$ and involucrin, was analyzed at mRNA (A) and protein (B) levels. For real time PCR analyses, total RNA was extracted from transfected cells and used for CDNA preparation. By the use of specific oligonucleotide primers, the relative levels of mRNA of Rac1, K14, K10, and involucrin were determined and expressed after normalization with the housekeeping gene 36B4 mRNA expression (A). For the Western blot analyses whole protein extracts from transfected cells were probed after electrophoresis and transfer with specific primary antibodies against Rac1, K14, K10 or involucrin (B). Beta actin levels were analyzed as loading control. The results are representative of three independent experiments.

\section{Rac1 Silencing and its Consecutive Effects on Keratinocyte Differentiation Are Not Accompanied by Any Change in Phosphorylation of Mitogen-Activated Protein Kinase (MAPK) Family Members-ERK1/2, p38, JNK1/2}

Since functional interactions between Rac1 and MAPK family members, ERK1/2, p38, and JNK1/2, have been reported in several systems different than keratinocytes [Coso et al., 1995; Minden et al., 1995; Frost et al., 1997], we examined the consequences of Rac1 silencing on the activation of these three signaling pathways in NHAK. For this purpose, protein extracts of keratinocytes treated with siRac1 or siRNA control were analyzed by Western blotting
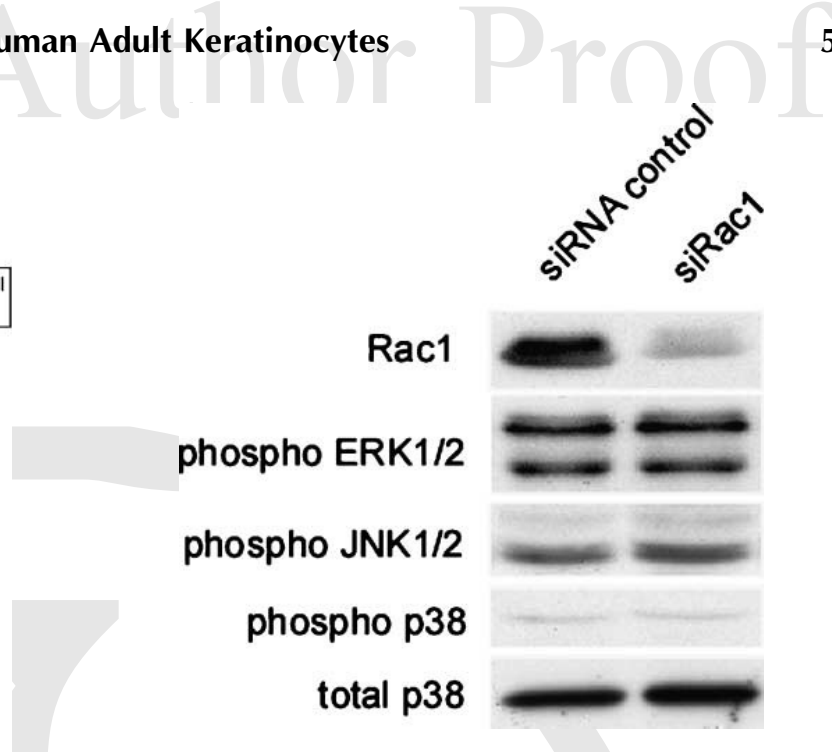

Fig. 4. Rac1 silencing does not affect the phosphorylation of MAPKs members-ERK $1 / 2$, p38, and JNK $1 / 2$ in NHAK. NHAK were transiently transfected with siRac1 or siRNA control as it was described in Materials and Methods. Three days after the transfection, the cells were harvested and whole protein extracts were analyzed by Western blotting with specific primary antibodies against Rac1, phospho-ERK1/2, phospho- and totalp38, or phospho-JNK1/2. Total p38 protein levels confirm the equivalent protein loading on the gel. Two independent experiments confirmed the results.

using specific antibodies against phospho ERK1/2, against phospho JNK1/2, or against phospho p38 and total p38 (Fig. 4): the inhibited Rac1 expression in keratinocytes does not influence the level of neither of the three phospho MAPKs, suggesting no interaction between Rac1 and the MAPKs in these conditions.

\section{In Human Keratinocytes, Rac1 Silencing Impedes Ser62/Thr58 Phosphorylation in c-myc}

Three days after transient transfection with siRac1, or siRNA control, NHAK were analyzed for both c-myc expression and phosphorylation on residues Ser62 and Thr58 by Western blotting and specific antibodies. The silencing of Rac1 does not alter expression of c-myc but induces a decreased Ser62/Thr58 phosphorylation (Fig. 5), suggesting that Rac1 is involved directly or indirectly in the regulation of this phosphorylation known to control myc stability and accumulation in several different cell types [Sears et al., 2000; Vervoorts et al., 2006].

\section{DISCUSSION}

Several recent studies have suggested that Rho family proteins, including Rac1, play crucial roles in regulation of mouse keratinocyte's 


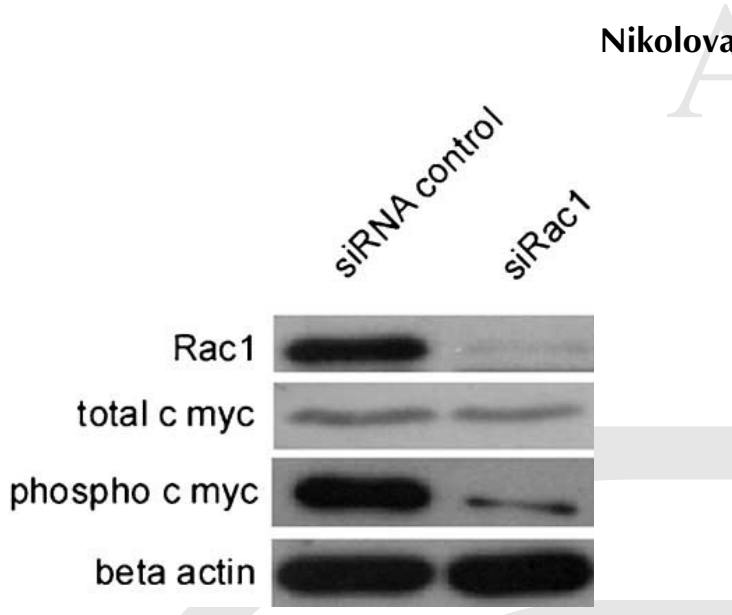

Fig. 5. Rac1 silencing inhibits Ser62/Thr58 phosphorylation of c-myc in NHAK. NHAK were transiently transfected with siRac1 or siRNA control (see Materials and Methods). Three days after transfection, the treated keratinocytes were harvested, their total protein content was extracted, and then analyzed by Western blotting with specific primary antibodies against Rac1, total c-myc and Ser62/Thr58 phosphorylated c-myc. Beta-actin was used as a loading control. Two independent experiments confirmed the results.

physiology, well beyond the establishment of cell-cell adhesion [Benitah et al., 2005; Grossi et al., 2005; Chrostek et al., 2006]. The main purpose of our current study was to assess in cultured human keratinocytes whether Rac1 functions in controlling growth/differentiation in this cell type and to compare our results with previous data obtained with other Rac1-related Rho family members, Rho-A, -B, and -C or with Rac1 itself in mouse mutants. For this study, we elected the RNA interference technology in order to get the most specific effects on cultured NHAK [Wang and Zheng, 2007].

Grossi and her colleagues treated growing mouse and human keratinocytes with exoenzyme C3, a toxin produced by Clostridium botulinum that selectively inhibits RhoA, -B, and $-\mathrm{C}$ but not Rac1 and found that this treatment does not affect the proliferative activity of the cells, but is sufficient to induce expression of differentiation markers (K1, K10, involucrin, filaggrin). Since a selective and potent inhibitor for Rac1 was not available, Benitah et al. [2005] and Chrostek et al. [2006] independently generated two different mice mutants with restricted deletion of the Rac1 gene in epidermis. The two studies however reported results with strikingly contrasting observations, probably depending on the strain (genetic background) of the analyzed mice or on the method used for Rac1 disruption. As Chrostek et al. [2006] discussed in their study,

one example of such a discrepancy has already been reported for differences in skin, brain and lung phenotypes in EGF receptor-deficient mice, depending on the strain of the analyzed animals [Sibilia et al., 1998]. According to Benitah et al. [2005], epidermal deletion of Rac1 causes increased proliferation of basal keratinocytes, followed by increased expression of the differentiation marker K10, mediated by induced c-myc expression and activity. Conversely according to Chrostek et al. [2006], keratinocyte-restricted deletion of rac1 gene in mutant mice does not affect the normal proliferation rate of basal keratinocytes, does not change the expression of differentiation-specific K10 and loricrin and does not alter c-myc expression.

In our in vitro study with human keratinocytes, we found that Rac1 silencing does not significantly influence the proliferative activity of the cells. Although Benitah et al. [2005] reported an increase of basal keratinocyte proliferation and some thickening of the Rac1 deficient epidermis, Chrostek et al. [2006] found no effect on epidermal proliferation in their Rac1-null mice.

Again in our in vitro study, we report that Rac1 suppression induces premature expression of the two differentiation makers we analyzed, K10 and involucrin. This is in contrast with the lack of effect on differentiation observed by Chrostek et al. [2006] in their Rac1deficient mice mutant. Thus, our results suggest that in human cultured keratinocytes Rac1 exerts negative control on the epidermal differentiation without influencing cell proliferation. Our results demonstrate similar effects of Rac1 inhibition with those established by Grossi et al. [2005] after inhibition of Rho A, B, and C.

The p38 MAPK family member is known to promote keratinocyte differentiation and apoptosis [Eckert et al., 2002]. Thus, we have investigated whether premature induction of differentiation markers after suppression of Rac1 could be mediated by activation of p38. However, our results have shown no influence of Rac 1 suppression on the activation of $\mathrm{p} 38$, or on the activation of neither of the two other MAPK family members, ERK1/2 and JNK1/2. This concurs with the mouse model of Chrostek et al. [2006] where epidermal deletion of Rac1 neither affects p38 or JNK activation.

Another discrepancy between the two mice models of Benitah et al. [2005] and Chrostek 
et al. [2006] concerns the effect of Rac1 deletion on the transcription factor c-myc. According to Benitah's study [2005], Rac1 sustains basal proliferation in keratinocytes through negative regulation of c-myc expression and activity. However according to Chrostek et al. [2006], keratinocytes-restricted Rac1 deletion does not influence c-myc expression. In our present study, we explored in cultured human keratinocytes the consequences of Rac1 silencing on c-myc expression and c-myc phosphorylation at its two N-terminal phosphorylation sites, Ser62/Thr58, and found that Rac1 suppression does not alter c-myc expression, but negatively regulates c-myc phosphorylation. Ser62/Thr58 phosphorylation has been previously found to be critical for determining the stability of c-myc. Phosphorylation of Ser62 is involved in stabilizing c-myc by increased half-life and activity. The phosphorylation of Thr58 depends on the prior phosphorylation of Ser62 [Vervoorts et al., 2006]. Thr58 phosphorylation promotes c-myc degradation through the ubiquitin/proteasome system, controlling the duration of c-myc activity. Ser62/Thr58 phosphorylation emerges as an important mechanism for posttranslational control of c-myc protein levels and activity [Sears et al., 2000]. In fibroblasts and in cell types different than keratinocytes, c-myc is detected at high levels of Ser62/Thr58 phosphorylation after growth stimulation, when c-myc protein plays critical role in promoting G1/S progression of the cell cycle [Berns et al., 1997; Liu et al., 2004; Murphy et al., 2004]. In contrast, in epidermal keratinocytes, the functions of c-myc have been reported to be not so simple and even contradictory. From one side, Benitah et al. [2005] and Murphy et al. [2005] found that c-myc activity promotes keratinocyte differentiation and keratinocytes stem cells proliferation is sustained by c-myc suppression. Conversely, Zanet et al. [2005] established in epidermal Myc knock-out (KO) mutant mice that a lack of c-myc causes premature differentiation-the markers of early terminal differentiation, $\mathrm{K} 1$ and $\mathrm{K} 10$ being extensively expressed in the basal layer of KO epidermis, where also later differentiation markers, involucrin and filaggrin, were also detected. Interestingly, keratinocytes of the basal layer continued to proliferate normally. A third bunch of data argues about pro-proliferative functions of c-myc in keratinocytes. It has been found that the inhibition of CaMK and MAPK by their selective pharmacological inhibitors (KN-62 and PD98059, respectively) inhibits c-myc activity and human keratinocyte proliferation [Praskova et al., 2002]. c-myc appears to gather signals from different transduction pathways which probably modulate its activity in different ways, explaining the observed contradictory effects of c-myc in epidermal keratinocytes.

In our study, we further observed that Rac1 suppression inhibits c-myc phosphorylation on Ser62/Thr58. This inhibition is concomitant with premature expression of differentiation markers but do not significantly influence the proliferative activity of the keratinocytes. These results concur with those of Zanet et al. [2005] who found that c-myc exhibits a negative effect of on the expression of differentiation markers in normal mouse epidermis but lacks any effect on basal keratinocytes proliferation.

Taken together, our results demonstrate that in regard of growth/differentiation in human keratinocytes, Rac1 exerts the same regulatory functions as was established for the other Rho family members, Rho-A, -B, and -C. Rho and Rac1 exert negative control on keratinocyte differentiation. Because Grossi et al. [2005] found in their study that Rho-A, -B, and -C exert the negative control on keratinocytes differentiation through activation of their effector protein, CRIK kinase, the involvement of CRIK kinase in the control exerted by Rac1 on keratinocyte differentiation should now be investigated, especially since an active mutant form of Rac1 has been found to increase CRIK kinase activity [Di Cunto et al., 1998]. Finally, it must be noticed that Grossi et al. [2005] have proposed that Rho/CRIK pathway is of significance in vivo under conditions such as wound healing, when the normal differentiation process is arrested and/or reversed. Hence, the very recently illustrated role of Rac1 in epidermal wound healing [Tscharntke et al., 2007] and the significant impairment of epidermal hyperproliferation during wound healing in absence of Myc [Zanet et al., 2005] concur to suggest that the functional interaction between Rac1 and c-myc might be required for epidermal hyperproliferation during wound-healing.

\section{ACKNOWLEDGMENTS}

We thank Dr. B. Bienfait (Clinique Saint-Luc, Bouge) for providing the normal skin samples. $\mathrm{EN}$ was holding a $\mathrm{PhD}$ fellowship for foreign 
students from the University of Namur (FUNDP). Financial support through FRFC grant 2.4506.01 to YP.

\section{REFERENCES}

Benitah $\underline{\mathrm{AS}}^{\mathrm{Q} 2}$, Frye M, Glogauer $\underline{\mathrm{XX}}^{\mathrm{Q3}}$, Watt MF. 2005. Stem cell depletion through epidermal deletion of Rac1. Science 309:933-935.

Berns K, Hijmans EM, Bernards R. 1997. Repression of c-Myc responsive genes in cycling cells caused G1 arrest trough reduction of cyclin $\mathrm{E} / \mathrm{CDK} 2$ kinase activity. Oncogene 15:1347-1356.

Braga VM, Machesky LM, Hall A, Hotchin NA. 1997. The small GTP-ases Rho and Rac are required for the establishment of cadherin-dependent cell-cell contacts. J Cell Biol 137:1421-1431.

Chrostek A, Wu X, Quondamatteo F, Hu R, Sanecka A, Niemann C, Langbein L, Haase I, Brakebusch C. 2006. Rac1 is crucial for hair follicle integrity but is not essential for maintenance of the epidermis. Mol Cell Biol 26:6957-6970.

Coso OA, Chiariello M, Yu JC, Teramoto H, Crespo P, Xu N, Miki T, Gutkind JS. 1995. The small GTP-binding proteins Rac1 and Cdc42 regulate the activity of the JNK/SAPK signalling pathway. Cell 81:1137-1146.

De Luca M, Pellegrini G, Zambruno G, Marchisio PC. 1994. Role of integrins in cell adhesion and polarity in normal keratinocytes and human skin pathologies. J Dermatol 21:821-828.

Di Cunto F, Calautti E, Hsiao J, Ong L, Topley G, Turco E, Dotto GP. 1998. Citron rho-interacting kinase, a novel tissue-specific ser/thr kinase encompassing the RhoRac-binding protein Citron. J Biol Chem 273:2970629711.

Eckert RL, Efimova T, Dashti SR, Balasubramanian S, Deucher A, Crish JF, Sturniolo M, Bone F. 2002. Keratinocyte survival, differentiation, and death: Many roads lead to mitogen-activated protein kinase. J Investig Dermatol Symp Proc 7:36-40.

Frost JA, Steen H, Shapiro P, Lewis T, Ahn N, Shaw PE, Cobb MH. 1997. Cross-cascade activation of ERKs and ternary complex factors by Rho family proteins. EMBO J 16:6426-6438.

Grossi M, Hiou-Feige A, Tommasi Di Vignano A, Calautti E, Ostano P, Lee S, Chiorino G, Dotto GP. 2005. Negative control of keratinocyte differentiation by Rho/CRIK signalling coupled with up-regulation of KyoT1/2 (FHL1) expression. PNAS 102:11313-11318.

Iizuka H, Takahashi H, Honma M, Ishida-Yamamoto A. 2004. Unique keratinization process in psoriasis: Late differentiation markers are abolished because of premature cell death. J Dermatol 31:271-276.
Liu J, Puscheck EE, Wang F, Trostinskaia A, Barisic D, Maniere G, Wygle D, Zhong W, Rings EH, Rappolee DA. 2004. Serine-threonine kinases and transcription factors active in signal transduction are detected at high levels of phosphorylation during mitosis in preimplantation embryos and trophoblast stem cells. Reproduction 128: 643-654.

Minden A, Lin A, Claret FX, Abo A, Karin M. 1995. Selective activation of the JNK signalling cascade and c-Jun transcriptional activity by the small GTPases Rac and Cdc42Hs. Cell 81:1147-1157.

Murphy LO, MacKeigan JP, Blenis J. 2004. A network of immediate early gene products propagates subtle differences in mitogen-activated protein kinase signal amplitude and duration. Mol Cell Biol 24:144-153.

Murphy MJ, Wilson A, Trumpp A. 2005. More than just proliferation: Myc function in stem cells. Trends Cell Biol 15:128-137.

Poumay Y, Herphelin F, Smits P, De Potter IY, Pittelkow MR. 1999. High cell density, phorbol ester and retinoic acid upregulate involucrin and downregulate suprabasal keratin 10 in autocrine cultures of human epidermal keratinocytes. Mol Cell Biol Res Commun 2:138-144.

Praskova M, Kalenderova S, Miteva L, Poumay Y, Mitev V. 2002. $\mathrm{Ca}(2+) /$ calmodulin-dependent protein kinase (CaM-kinase) inhibitor KN-62 suppresses the activity of mitogen-activated protein kinase (MAPK), c-myc activation and human keratinocyte proliferation. Arch Dermatol Res 294:198-202.

Rao KS, Babu KK, Gupta PD. 1996. Keratins and skin disorders. Cell Biol Int 20:261-274.

Sears R, Nuckolls F, Haura E, Taya Y, Tamai K, Nevins JR. 2000. Multiple Ras-dependent phosphorylation pathways regulate Myc protein stability. Genes Dev 14: 2501-2514.

Sibilia M, Steinbach JP, Stingl L, Aguzzi A, Wagner EF. 1998. A strain-independent postnatal neurodegeneration in mice lacking the EGF receptor. EMBO J 17:719-731.

Tscharntke M, Pofahl R, Chrostek-Grashoff A, Smyth N, Niessen C, Niemann C, Hartwig B, Herzog V, Klein HW, Krieg T, Brakebusch C, Haase I. 2007. Impaired epidermal wound healing in vivo upon inhibition or deletion of Rac1. J Cell Sci 120:1480-1490.

Vervoorts J, Lüscher-Firzlaff J, Lüscher B. 2006. The ins and outs of myc regulation by posttranslational mechanisms. J Biol Chem 281:34725-34729.

Wang L, Zheng Y. 2007. Cell type-specific functions of Rho GTPases revealed by gene targeting in mice. Trends Cell Biol 17:58-64.

Zanet JP, Pibre S, Jacquet C, Ramirez A, de Alboran IM, Gandarillas A. 2005. Endogenous Myc controls mammalian epidermal cell size, hyperproliferation, endoreplication and stem cell amplification. J Cell Sci 118: 1693-1704.

Q1: Please check the corresponding author address.

Q2: Please check the authors name.

Q3: Please provide the first name. 